\title{
p53 protein expression in synchronously occurring dedifferentiating stages of thyroid cancer in a patient with neurofibromas: A case report
}

\author{
GEORGIOS BOUTZIOS $^{1}$, ELENI PAPAOICONOMOU ${ }^{1}$, ANDREAS PIKOULIS ${ }^{2}$, \\ KONSTANTINOS NASTOS ${ }^{2}$, DESPOINA POULOUDI ${ }^{3}$, ANASTASIA PIKOULI ${ }^{2}$, \\ ELENI KOUKOULIOTI $^{1}$, ANDREAS LAZARIS ${ }^{3}$ and EMMANOUIL PIKOULIS ${ }^{2}$ \\ ${ }^{1}$ Department of Pathophysiology, Laiko University Hospital, Medical School,
} National and Kapodistrian University of Athens, 11527 Athens; ${ }^{2}$ Third Department of Surgery, Attikon University Hospital, National and Kapodistrian University of Athens, 12462 Athens; ${ }^{3}$ First Department of Pathology Medical School, National and Kapodistrian University of Athens, 11527 Athens, Greece

Received April 16, 2020; Accepted June 15, 2021

DOI: $10.3892 / \mathrm{mco} .2021 .2362$

\begin{abstract}
Poorly differentiated thyroid carcinoma (PDTC) and anaplastic thyroid carcinoma (ATC) have been hypothesized to arise from well-differentiated thyroid carcinoma (WDTC) due to frequently reported synchronous and metachronous occurrence. Loss of normal p53 function has been implicated in this dedifferentiation process. The current case report presents a 60 -year-old male with multiple neurofibromas who underwent total thyroidectomy due to multiple palpable thyroid nodules. Histopathological examination revealed three foci of predominantly papillary, but also follicular carcinoma growth pattern, and two lesions with histological features of insular and trabecular variant, with the larger one showing foci of anaplastic transition. Nuclear p53 protein accumulation, corresponding to mutant abnormally stabilized $\mathrm{p} 53$, was higher in more aggressive variants compared with WDTC. The somatic molecular events and downstream pathways of this dedifferentiation course have not been unraveled yet. The present case report demonstrated the simultaneous presence of three divergent histological subtypes in a single thyroid gland, with progressive enhancement of nuclear $\mathrm{p} 53$ protein expression, associated with mutant $\mathrm{p} 53$ protein, in the more aggressive variants. This is a rare case of progressive enhancement of mutant nuclear $\mathrm{p} 53$ protein expression in multifocal thyroid tumor areas consisting of WDTC, PDTC and ATC histological types, highlighting the possibility that WDTC can
\end{abstract}

Correspondence to: Professor Konstantinos Nastos, Third Department of Surgery, Attikon University Hospital, National and Kapodistrian University of Athens, 1 Rimini Street, Chaidari, 12462 Athens, Greece

E-mail: kosnastos@med.uoa.gr

Key words: papillary carcinoma, follicular carcinoma, poorly differentiated carcinoma, anaplastic carcinoma, p53 progress to PDTC and then ATC through an intricate procedure, involving loss of normal p53 function.

\section{Introduction}

Poorly differentiated thyroid carcinoma (PDTC) is a clinically and molecularly inchoate histologic variant of thyroid carcinoma. PDTC occupies an intermediate position between well-differentiated thyroid carcinoma (WDTC) and anaplastic thyroid carcinoma (ATC) with regard to both histological features and biologic aggressiveness and is often associated with locoregional recurrence and distant disease leading to compromised survival $(1,2)$. PDTC was originally classified as a distinct clinicopathologic variant in 2004 by the World Health Organization (WHO), but it was not until 2006 that more stringent criteria, known as the 'Turin criteria', were established to allow diagnosis standardization $(2,3)$. Turin proposal stated that PDTC diagnosis should be rendered on histologic specimens demonstrating solid/trabecular/insular growth pattern, absence of conventional nuclear features of PTC, and at least one of the following features: Convoluted nuclei, necrosis or mitotic index $\geq 3 / 10$ high power fields (HPFs) (2-4).

Although PDTC has distinct diagnostic criteria and can develop de novo, in a significant proportion of patients, PDTC-like features may occur simultaneously with follicular, papillary or anaplastic carcinoma stressing the possibility that PDTC may arise by dedifferentiation of pre-existing WDTC $(5,6)$. The mutational events that orchestrate this malignant progression are ill-defined. Genetic alterations frequently detected in PDTC include TP53, RAS, BRAF and CTNNB1 genes $(7,8)$.

Environmental factors, such as iodine deficiency and prior neck irradiation, have been implicated in the pathogenesis of PDT (9). In chorus with other thyroid carcinoma subtypes, PDTC demonstrates a female predominance (9).

TP53 gene encodes a nuclear transcription factor that regulates cell cycle by inducing arrest at the G1/S check point and promotes apoptosis, hence functioning as a tumor suppression 
gene. Inactivation of the p53 gene has often been reported in undifferentiated thyroid carcinomas (10). Wild type p53 is an unstable protein which is present at very low cellular levels due to murine double minute gene 2 (MDM2)-mediated degradation by cytoplasmic and nuclear proteasomes (11). On the contrary mutated counterpart is resistant to MDM2-mediated degradation, resulting in abnormal stabilization and immunohistochemically detectable protein accumulation (12).

The aim of this case report presentation is to clearly depict and confirm the hypothesis of the natural progression of WDTC to ATC, showing the progression of tumor dedifferentiation in a single patient, with progressive loss of p53 expression from the well differentiated to the dedifferentiated components, confirming the hypothesis that p53 dysregulation may contribute to the natural history of the disease.

\section{Case report}

A 60-year-old man presented with a palpable anterior neck mass, which was firm and fixed to underlying structures. The mass caused mild compressive symptoms including neck fullness and dysphagia. The patient also manifested multiple cutaneous lesions with histopathologic features compatible with neurofibroma. He did not fulfill though at least one more of the seven cardinal diagnostic criteria for von Recklinghausen disease, as he did not have freckling of the groin or the axilla, café-au-lait macules, skeletal abnormalities, Lisch nodules or a first-degree relative with the disease. He refused imaging control to exclude an optic glioma, although optic fields were normal with finger confrontation test. He also refused mutational analysis. The diagnosis of von Recklinghausen disease could therefore not be established. Cervical radiation exposure was not elicited from past history. Iodine status did not need to be evaluated, since the patient lives in an iodine replete country.

The patient's medical record included hypercholesterolemia, psychotic disorder, posttraumatic acute subdural hematoma and nasal polyps. Family history was unremarkable, except from a 46-year-old sister with breast cancer and total thyroidectomy due to multinodular goiter.

Hormonal evaluation was conducted to evaluate thyroid function: TSH was $2.5 \mu \mathrm{IU} / \mathrm{ml}$ (normal values 0.27-4.2) and calcitonin $4.2 \mathrm{pg} / \mathrm{ml}$ (normal values $<10 \mathrm{pg} / \mathrm{ml}$ ). The patient was also subjected to $24 \mathrm{~h}$ urine collection to rule out the presence of a pheochromocytoma, on grounds of unproven von Recklinghausen disease, at the prospect of surgery: Vanillylmandelic acid (VMA) $2.1 \mu \mathrm{g} / 24 \mathrm{~h}$ (normal values 1-11), catecholamines $220 \mu \mathrm{g} / 24 \mathrm{~h}$ (normal values 80-515), metanephrines $108 \mu \mathrm{g} / 24 \mathrm{~h}$ (normal value 52-341), adrenaline $6 \mu \mathrm{g} / 24 \mathrm{~h}$ (normal values 4-25), noradrenaline $46.1 \mu \mathrm{g} / 24 \mathrm{~h}$ (normal values 20-105), dopamine $168.6 \mu \mathrm{g} / 24 \mathrm{~h}$ (normal values 60-440).

Ultrasound revealed a $5.84 \times 2.81 \mathrm{~cm}$ hypoechogenic nodule on the left lobe with peripheral vascularity, which comprised a $2.24 \times 1.28 \mathrm{~cm}$ area with suspicious calcifications. Three smaller hypoechogenic nodules with peripheral vascularity, measuring 3.26, 3.18 and $0.83 \mathrm{~cm}$ respectively, were also observed on the same lobe. On the right lobe three isoechogenic nodules with cystic areas and no remarkable vascularity, dimensions 2.19, 1.47 and $0.73 \mathrm{~cm}$, were found. No suspicious lymph nodes were detected by ultrasound.

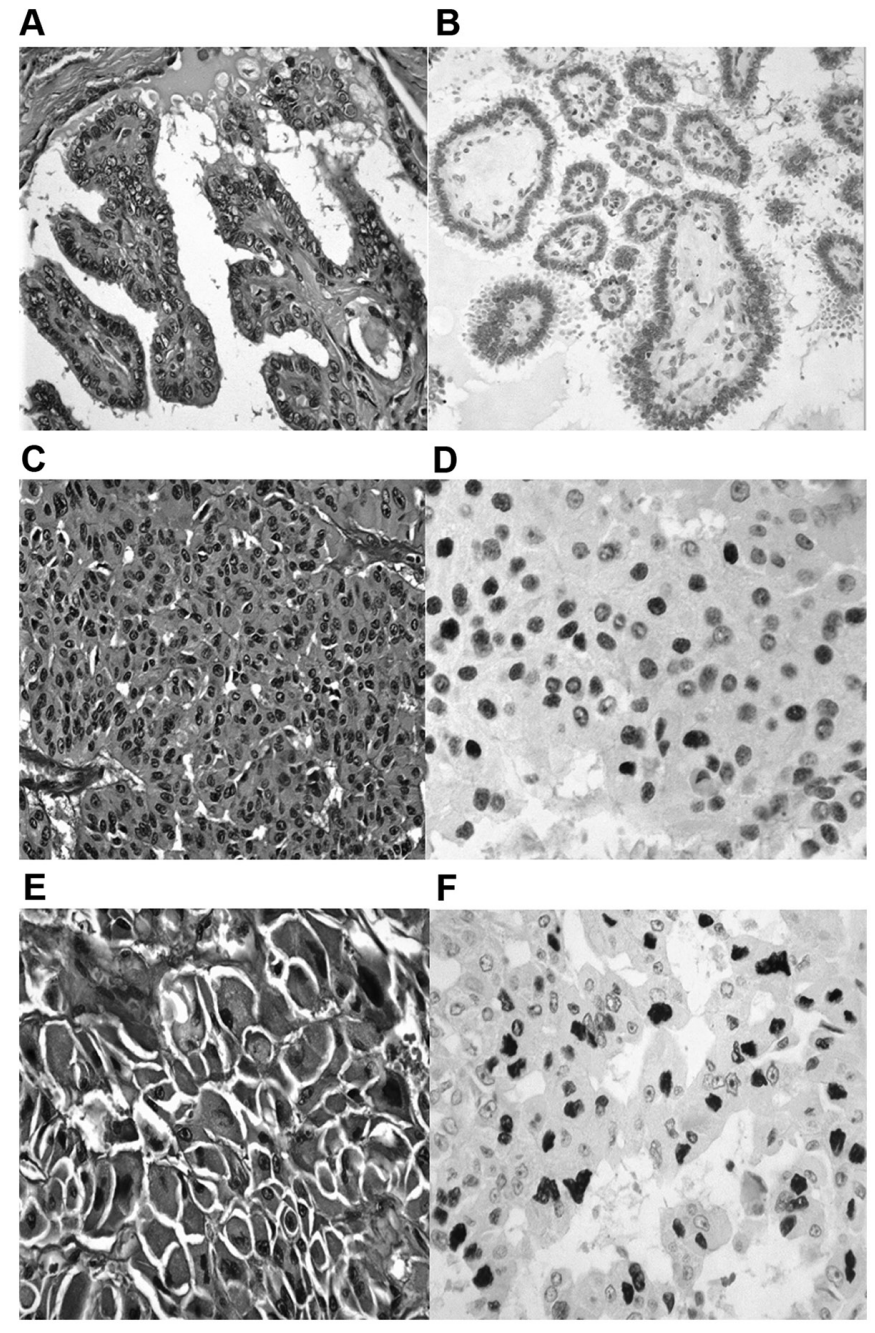

Figure 1. (A) Well differentiated papillary carcinoma (H\&E; magnification, $\mathrm{x} 400$ ). (B) Weak nuclear p53 protein expression in well differentiated papillary carcinoma (magnification, $\mathrm{x} 400$ ). (C) Poorly differentiated tumor area with insular growth pattern (H\&E; magnification, x400). (D) Diffuse, moderate to strong nuclear p53 protein accumulation (magnification, $\mathrm{x} 400$ ). (E) Tumor area with tendency for dedifferentiation and nuclear anaplasia (H\&E; magnification, x400). (F) Diffuse strong nuclear p53 protein accumulation in dedifferentiated area (magnification, $\mathrm{x} 400$ ). H\&E, hematoxylin and eosin.

Cytological examination of a fine needle aspiration (FNA) biopsy specimen was omitted from the diagnostic workup due to patient refusal, and consequently the patient underwent total thyroidectomy. Postoperative course was uneventful. The specimens underwent histopathological examination by classic $\mathrm{H} \& \mathrm{E}$ staining and immunohistochemical detection of p53 protein, as previously described (13). Briefly, H\&E staining was performed according to routine hospital procedures, while p53 staining was performed using a mouse monoclonal anti-p53 antibody (1:3,000; DO-7; Dako) as the primary antibody. The staining intensity was graded as negative, weak, moderate and strong. Histopathological examination revealed three foci at the right lobe, dimensions $0.6,0.8$ and $2.4 \mathrm{~cm}$ respectively, which showed predominantly papillary but also follicular growth pattern with infiltrating margins and foci of extrathyroidal extension.

At the left lobe two lesions of 6.0 and $6.5 \mathrm{~cm}$ with PDTC-like histologic features of insular and trabecular 
variant of PDTC were described. The tumors were composed of small uniform cells and of cells with large irregular nuclei, with partially acidophil characteristics, and were surrounded by fibrous stroma. The larger tumor showed foci of anaplastic transition, with diffuse growth pattern, nuclear pleomorphism and areas of necrosis. Both tumors invaded thyroid capsule and displayed extrathyroidal extension reaching the inked surgical margins. Vascular emboli were also identified. A dissected isthmic lymph node was free of disease.

WDTC carcinoma demonstrated weak nuclear p53 protein expression (Fig. 1A and B). PDTC appeared with diffuse, moderate to strong nuclear $\mathrm{p} 53$ protein accumulation (Fig. 1C and D). ATC showed diffuse, strong nuclear p53 protein accumulation (Fig. 1E and F). The patient was referred for radioiodine remnant ablation.

\section{Discussion}

PDTC and ATC have been conjectured to arise from WDTC due to frequently reported synchronous and metachronous occurrence. The somatic molecular events and downstream pathways of this dedifferentiation course have not been unraveled yet. In this paper we demonstrate the simultaneous presence of the three divergent histological subtypes in a single thyroid gland, with progressive enhancement of nuclear p53 protein expression, correlating with mutant p53 protein, in the more aggressive variants.

PDTC is a rare thyroid carcinoma histotype that occupies an intermediate position between WDTC and ATC in regards of both morphological features and biologic behavior $(1,2)$. PDTC was initially designated as a distinct entity in 2004 by $\mathrm{WHO}$, but more refined criteria were established in the 2006 Turin proposal (2-4). In juxtaposition to WDTH, which usually has an indolent course, PDTC diagnosis signals a steep increase in mortality and accounts for a disproportionate number of deaths due to non-ATC (9).

The coexistence of WDTC or ATC with PDTC-like tumor components has frequently been observed, suggesting that PDTC could represent a bridge in the stepwise dedifferentiation from WDTC to ATC (14). Although the molecular pathways underlying this intricate procedure have not been elucidated yet, loss of p53 tumor suppressor gene has been assumed to convey to this anaplastic transformation, since p53 is well expressed in WDTC but abated in PDTC and ATC (15).

Genetic analysis of thyroid malignancies has shown varying correlations of specific mutations with tumor aggressiveness. Although rearrangement of the RET proto-oncogene are frequent even in the early stages of thyroid tumorigenesis, RAS oncogene family mutations and BRAF600 gene mutations have failed to date to show a distinct prognostic significance for recurrence and overall survival. In the other hand, mutations in the TERT promoter gene (especially in combination with BRAF600 gene mutations) and p53 tumor suppressor gene mutations, have been associated with poor prognosis and have been implicated in the progression of WDTC to dedifferentiation (16). In detail, mutations in p53 gene leads to either the expression of mutant protein or to complete absence of expression. These mutations are seen in up to $25 \%$ of patients with
PDTC and in over $60 \%$ of patients with ATC (17). However, various studies have shown mutations in well differentiated tumors as well $(18,19)$. This reveals that p53 mutation is an early event in the sequence of tumor dedifferentiation and transformation to ATC.

There is no explicit consensus regarding the optimal management of PDTC, partially due to its infrequent occurrence and the previous heterogeneity of inclusion criteria $(1,20,21)$. The mainstay of treatment usually comprises total thyroidectomy plus regional lymph dissection when required whereas the efficacy of other modalities, such as radioactive iodine ablation (RAI), external beam radiation therapy and chemotherapy is still questionable (22). Although RAI treatment is commonly implemented, PDTC may exhibit decreased radio avidity and thus be refractory $(6,22)$.

Finally, although our patient did not typically fulfill the diagnostic criteria for von Recklinghausen disease, the coexistence of neurofibromas is intriguing due to the scarcity of reported coexistence of thyroid carcinoma with neurofibromatosis in the literature (23-25).

Both PDTC and ATC demonstrate aggressive behavior and forecast a robust increase in coherent mortality. It is consequently of key interest to illuminate the mechanisms of the dedifferentiation process. Herein, we present a rare case of gradual enhancement of nuclear mutated p53 protein expression in multifocal thyroid tumor areas consisting of WDTC, ATC histology and PDTC-like features. This observation strengthens the postulation that ATC and PDTC could arise from progenitor foci of WDTC through a multifactorial process involving loss of normal p53 function.

\section{Acknowledgements}

Not applicable.

\section{Funding}

No funding was received.

\section{Availability of data and materials}

Data sharing is not applicable to this article, as no datasets were generated or analyzed during the current study, as this is a report of an interesting case. Patient records are available upon request from the corresponding author.

\section{Authors' contributions}

GB, KN, EK and EPi contributed to conception and design of the manuscript. EPa, AL, DP, AnaP and AndP acquired, analyzed and interpreted the data, and drafted the manuscript. $\mathrm{GB}, \mathrm{KN}$ and EPi revised the manuscript. DP, KN and EK confirm the authenticity of all the raw data. All authors have read and approved the final version of this manuscript.

\section{Ethics approval and consent to participate}

No ethics approval was obtained as the patient received standard treatment. Consent for treatment was obtained by the patient per protocol. 


\section{Patient consent for publication}

The patient has provided written informed consent for the publication of this case report.

\section{Competing interests}

The authors declare that they have no competing interests.

\section{References}

1. DeLellis RA, Lloyd VR, Heitz PU and Eng C (eds): World Health Organization Classification of Tumours: Pathology and Genetics of Tumours of Endocrine Organs. IARC Press, Lyon, 2004

2. Sadow PM and Faquin WC: Poorly differentiated thyroid carcinoma: An incubating entity. Front Endocrinol (Lausanne) 3: 77, 2012.

3. Tallini G: Poorly differentiated thyroid carcinoma. Are we there yet? Endocr Pathol 22: 190-194, 2011.

4. Volante M, Collini P, Nikiforov YE, Sakamoto A, Kakudo K, Katoh R, Lloyd RV, LiVolsi VA, Papotti M, Sobrinho-Simoes M, et al: Poorly differentiated thyroid carcinoma: The turin proposal for the use of uniform diagnostic criteria and an algorithmic diagnostic approach. Am J Surg Pathol 31: 1256-1264, 2007.

5. Garcia-Rostan G and Sobrinho-Simões M: Poorly differentiated thyroid carcinoma: An evolving entity. Diagnostic Histopathol 17: 114-123, 2011.

6. Volante $\mathrm{M}$ and Papotti M: Poorly differentiated thyroid carcinoma: 5 years after the 2004 WHO classification of endocrine tumours. Endocr Pathol 21: 1-6, 2010.

7. Eloy C, Ferreira L, Salgado C, Soares P and Sobrinho-Simões M: Poorly differentiated and undifferentiated thyroid carcinomas. Turk Patoloji Derg 31 (Suppl 1): S48-S59, 2015.

8. Omur O and Baran Y: An update on molecular biology of thyroid cancers. Crit Rev Oncol Hematol 90: 233-252, 2014.

9. Walczyk A, Kowalska A and Sygut J: The clinical course of poorly differentiated thyroid carcinoma (insular carcinoma)-own observations. Endokrynol Pol 61: 467-473, 2010.

10. Quiros RM, Ding HG, Gattuso P, Prinz RA and Xu X: Evidence that one subset of anaplastic thyroid carcinomas are derived from papillary carcinomas due to BRAF and p53 mutations. Cancer 103: 2261-2268, 2005.

11. Levine AJ: p53, the cellular gatekeeper for growth and division. Cell 88: 323-331, 1997.

12. Midgley CA and Lane DP: p53 protein stability in tumour cells is not determined by mutation but is dependent on Mdm2 binding. Oncogene 15: 1179-1189, 1997.

13. Yemelyanova A, Vang R, Kshirsagar M, Lu D, Marks MA, Shih IM and Kurman RJ: Immunohistochemical staining patterns of p53 can serve as a surrogate marker for TP53 mutations in ovarian carcinoma: An immunohistochemical and nucleotide sequencing analysis. Mod Pathol 24: 1248-1253, 2011.
14. Lam KY, Lo CY, Chan KW and Wan KY: Insular and anaplastic carcinoma of the thyroid: A 45-year comparative study at a single institution and a review of the significance of p53 and p21. Ann Surg 231: 329, 2000.

15. McIver B, Hay ID, Giuffrida DF, Dvorak CE, Grant CS, Thompson GB, van Heerden JA and Goellner JR: Anaplastic thyroid carcinoma: A 50-year experience at a single institution. Surgery 130: 1028-1034, 2001.

16. Penna GC, Vaisman F, Vaisman M, Sobrinho-Simoes M and Soares P: Molecular markers involved in tumorigenesis of thyroid carcinoma: Focus on aggressive histotypes. Cytogenet Genome Res 150: 194-207, 2016.

17. Manzella L, Stella S, Pennisi MS, Tirro E, Massimino M, Romano C, Puma A, Tavarelli M and Vigneri P: New insights in thyroid cancer and p53 family proteins. Int J Mol Sci 18: 1325, 2017.

18. Marcello MA, Morari EC, Cunha LL, De Nadai Silva AC, Carraro DM, Carvalho AL, Soares FA, Vassallo J and Ward LS: P53 and expression of immunological markers may identify early stage thyroid tumors. Clin Dev Immunol 2013: 846584, 2013.

19. Shin MK, Kim JW, Min SK, Lee DJ, Kim JH, Lee SC, Chung BW and Ju YS: Associations of the BRAF (V600E) mutation and p53 protein expression with clinicopathological features of papillary thyroid carcinomas patients. Oncol Lett 10: 1882-1888, 2015.

20. Sanders EM Jr, LiVolsi VA, Brierley J, Shin J and Randolph GW: An evidence-based review of poorly differentiated thyroid cancer. World J Surg 31: 934-945, 2007.

21. Collini P, Mattavelli F, Pellegrinelli A, Barisella M, Ferrari A and Massimino M: Papillary carcinoma of the thyroid gland of childhood and adolescence: Morphologic subtypes, biologic behavior and prognosis: A clinicopathologic study of 42 sporadic cases treated at a single institution during a 30-year period. Am J Surg Pathol 30: 1420-1426, 2006.

22. Hannallah J, Rose J and Guerrero MA: Comprehensive literature review: Recent advances in diagnosing and managing patients with poorly differentiated thyroid carcinoma. Int J Endocrinol 2013: 317487, 2013.

23. Hashiba T, Maruno M, Fujimoto Y, Suzuki T, Wada K, Isaka T, Izumoto $\mathrm{S}$ and Yoshimine T: Skull metastasis from papillary thyroid carcinoma accompanied by neurofibromatosis type 1 and pheochromocytoma: Report of a case. Brain Tumor Pathol 23: 97-100, 2006.

24. Kim BK, Choi YS, Gwoo S, Park YH, Yang SI and Kim JH: Neurofibromatosis type 1 associated with papillary thyroid carcinoma incidentally detected by thyroid ultrasonography: A case report. J Med Case Rep 6: 179, 2012.

25. Koksal Y, Sahin M, Koksal H, Esen H and Sen M: Neurofibroma adjacent to the thyroid gland and a thyroid papillary carcinoma in a patient with neurofibromatosis type 1: Report of a case. Surg Today 39: 884-887, 2009. 